\title{
Effects of the Metropolitan Civil Resilience Program: Comparison of the SAT Resilience Program and an Exercise Program
}

\author{
Yoko Kobayashi $^{1}$, Tsunetsugu Munakata ${ }^{2}$ \\ ${ }^{1}$ Department of Risk Engineering, Graduate School of Systems, and Information Engineering, 1-1-1 Tennodai Tsukuba Ibaraki, 305- \\ 8577, Japan \\ ${ }^{2}$ Professor Emeritus of University of Tsukuba, 1-1-1 Tennodai Tsukuba Ibaraki, 305-8577, Japan
}

\begin{tabular}{l} 
A R T I C L E I N F O \\
\hline Article history: \\
Received: 12 December, 2016 \\
Accepted: 30 January, 2017 \\
Online: 25 February, 2017 \\
\hline
\end{tabular}

Keywords:

resilience

self-image

SAT (Structured Association

Technique)

\begin{abstract}
A B S T R A C T
Purpose: To conduct preliminary research for developing a program to enhance resilience, a topic that has generated attention of late.

Methods: We compared the Structured Association Technique (SAT) resilience program and an exercise program. The citizen's lecture on stress management occurred 1 month after East Japan Earthquake, and the intervention was carried out in the afternoon of the same day. Participants could choose to join SAT (an imagery therapy to improve the selfimage using associative images) resilience program (17 people) or healthy exercise program (Shakkiri gymnastics program; 9 people). They were metropolitan area residents, including workers and families. Participants filled in the checklist before the intervention, immediately after the intervention, and 1 month later. The saliva of all participants was extracted to assess SIgA as an immunity index before and immediately after the intervention. We compared the SAT group (9 females, age 34-60) and the exercise group (9 females, age 32-57).

Results: Compared to the exercise group, mental health indices such as self-solving and significantly improved in the SAT group. However, this does not suggest that there was no improvement in the exercise group. After intervention, in the exercise group, SIgA in saliva and depression improved. In the group, the immunity index shown in SIgA in the saliva, the mental health index as measured by state anxiety and depression, the self-image index as measured by self-affirmation, problem-solving, moral independence, and perceived family support all significantly improved.

Considerations: It appears that, through the change in self-image, resilience in daily life was promoted by the resilience program.
\end{abstract}

\section{Introduction}

The Japanese economy grew steadily before the 'Bubble economy' collapsed in the early 1990s. However, during the 1990s, it stopped growing. This situation induced extreme stress in society, leading to worsened mental health. Accordingly, suicides increased starting in the late $90 \mathrm{~s}$. Since 1998, there have been over 30,000 suicides over 14 years [1]. According to a survey by the Metropolitan Police Department, among cases with a document clearly indicating the cause of the suicide, about $47 \%$ were preceded by health problems, including mental health. In response,

Yoko Kobayashi, 1-1-1 Tennodai Tsukuba Ibaraki, 305-8577, Japan,

Email: s1630160@u.tsukuba.ac.jp

www.astesj.com

https://dx.doi.org/10.25046/aj020201 what is required of us is neither healing nor escaping from stress [2]. Rather, what is required is enhancing resilience to stress and learning from it, and then proactively changing the situation.

\subsection{Resilience}

The concept of resilience has drawn recent attention for various reasons. Resilience refers to the ability to recover from stress and fight against stress to improve the situation. Resilience is a term derived from physics originally used to reflect 'elasticity and repulsion.' In the 1970s, the study of resilience began in child psychiatry. Those with a healthy personality, despite having an adverse childhood, were considered highly resilient [3]. The study 


\section{Y. Kobayashi et al. / Advances in Science, Technology and Engineering Systems Journal Vol. 2, No. 2, 1-6 (2017)}

of resilience has now spread to recovery from war experience and post-traumatic stress disorder (PTSD) resulting from natural disasters like earthquakes and typhoons. It refers not only to recovery of normal mental health status, but the ability to fight against stresses, overcome, learn, and then change the situation [47].

How a person recognizes a stressful situation will in part determine whether the resulting state is despair or growth. Selfimage, that is, how one thinks and feels about the self, is a critical point here [8].

Munakata suggests that "self-image is the immobilized past memory of experience with self-interpretation and the memory synchronized with the self" and "a belief of self-image is named self-image script." He says that self-image determines the perspective for interpreting a stressful situation. High resilience to one's self-image is associated with high self-affirmation [9], problem-solving [10], self-expression, independence [11], and reduced depression [12].

\section{Methods}

\subsection{Recruitment of participants}

Participants were recruited from a metropolitan area, especially around Bunkyou-ku in Tokyo, and an announcement was created in some local newspapers. The citizen lecture on stress management was carried out at public facility A in Bunkyou-ku, Tokyo, 1 month after the Great East Japan Earthquake. There were 56 participants. After the lecture, we introduced the SAT resilience program and the Shakkiri exercise program as stress management programs. We asked participants to join the program in advance, and 55 participants were pre-registered on March 11, 2011. The intervention seminars took place in conference rooms A and B in the same public facility from 13:00 to 15:00.

\subsection{Intervention subjects}

There were 17 participants ( 3 male, 14 female) in the SAT resilience program (SAT group), and 9 (9 female) in the exercise program (Shakkiri exercise). Participants ranged from 21 to 78 years old, and participation rate from the civic lecture was $47.3 \%$. Participants selected one of the stress management programs.

\subsection{Invention protocol}

\section{Intervention preparation $(30 \mathrm{~min})$}

First, all subjects assembled in conference room A. We announced a brief description of the programs and asked the subjects to sign a consent form. Second, centrifuge tubes were given to each subject, and their saliva was collected. Each participant then answered an anonymous psychological questionnaire with a unique number. Collected saliva samples were closed and stored in a polystyrene foam box with ice packs box immediately to be sent for professional biochemical examination (SRL Co., Ltd) on the same day. Each subject had two centrifuge tubes for saliva collection and signed with their unique number and name by Katakana for saliva examination. We marked the collected centrifuge tubes to indicate pre-/post-intervention when tubes were collected. Immediately after the seminar, the box was sent for saliva examination. Delivery was specified for the next day.

2. SAT resilience program (1 hour)
The SAT resilience improvement sheet and the surrogate facial representative list were given to subjects in the conference room A. SAT therapy developer lectured on the SAT resilience program with these documents. He demonstrated image work modeling exercise with the resilience improvement sheet, and later, all subjects practiced the image work modeling exercises.

\section{Shakkiri exercise program (1 hour)}

We instructed subjects to arrive with exercise clothes. The exercise program called Shakkiri exercise was demonstrated by its developer in conference room B. First, the effects of Shakkiri exercise were described, which include releasing shoulder stiffness, leading to mental and physical refreshment. Following the instructions, subjects sat on chairs and practiced Shakkiri exercise with music.

\section{Psychological test and saliva collection (30 min)}

Both groups gathered again in conference room A after program completion.

Centrifuge tubes were handed out to each subject and their saliva was collected. Participants then answered the psychological questionnaire using their unique number, and no name was signed. Collected saliva was stored and delivered in the same way as in pre-intervention.

5. Collecting the questionnaire at 1-month follow-up

The same questionnaires were administered to subjects with a reply envelope to return the papers 1 month post-intervention. We sent a reminder to improve response rates.

\section{Ethical considerations}

We explained to participants orally and in writing that they would not be personally identified, and that the participation would be of their own free will. Approval was obtained from the Ethical Committee of the Graduate School of Comprehensive Human Sciences, University of Tsukuba.

7. Contents of the psychological questionnaire

a. Mental Resilience Scale (21 items, 63 points) [13]

b. Self-Affirmation Scale (10 items, 20 points) [14]

c. Self-Representation Behavioral Scale (10 items, 20 points) [14]

d. Problem Solving Behavioral Scale (10 items, 20 points) [15]

e. Mental Independence Behavioral Scale (10 items, 15 points) [15]

f. Mental Support from Family Scale (10 items, 10 points) [16]

g. Mental Support from Others Scale (10 items, 10 points) [17]

h. Self-Rating Depression Scale (SDS; 20 items, 80 points) [18] [19]

i. State-Trait Anxiety Inventory (STAI; 20 items, 80 points) [20] [21]

j. Face sheet items

Age, sex, occupation, counseling experience, and existence of present mental stress.

\subsection{Intervention details}




\section{SAT resilience program}

SAT therapy assumes that resilience is increased by belief in one's self-image, representing the self without others' feelings or thoughts, and active problem solving. There is a structured selfimage sheet based on the theoretical assumptions of SAT therapy. Participants were guided individually to follow the instructions with the self-image sheet. They created imaginary siblings and children with surrogate facial representatives. They were advised to look at the surrogate facial representatives daily.

\section{Shakkiri exercise program}

After warming up, participants stretched their bodies with light music while sitting on a chair. Rhythmic motion activates secretion of serotonin, leading to mental stability [22]. For cool down, participants were sprinkled with a scent and they relaxed their body with eyes closed. This program is intended to relax the body and soul. They were advised to practice Shakkiri exercise daily.

\subsection{Hypothesized intervention effects}

For the SAT resilience program, we hypothesized that intervention effects would show improved anxiety, depression, and increased salivary SIgA at post-intervention. Further, selfimage (script) would show improved self-representation, mental independence, self-affirmation, and problem-solving behavior. Depression in particular would decrease over 1 month. On the other hand, effects of Shakkiri exercise would include improved anxiety, depression, and increased salivary SIgA. However, selfimage factors are not hypothesized to change. Further, depression would not continue decreasing over 1 month.

\subsection{Analysis}

SPSS (version 19.0) was used for statistical analysis. Cronbach's alpha was computed to test reliability. Correlations were estimated using Spearman's rank-order correlation to study criterion-related validity based on SDS as the external criterion. Pre-/post-intervention results for psychological measured were measured using the Mann-Whitney U-test. For the 1-month follow-up, we used the Wilcoxon signed-rank test, with Bonferroni correction after the Friedman test.

Table 1 . Reliability and validity of each scale

\begin{tabular}{|c|c|c|c|c|c|}
\hline \multirow{2}{*}{ 9tiecte } & & \multicolumn{2}{|c|}{$\begin{array}{c}\text { Reliability } \\
\text { Cronbach's alpha a) }\end{array}$} & \multicolumn{2}{|c|}{$\begin{array}{c}\text { Criterion-related validity b) } \\
\text { Depression SOS }\end{array}$} \\
\hline & & 6el & 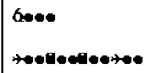 & 6ed & $\begin{array}{l}\text { 6eee } \\
\text { teellectlecteres }\end{array}$ \\
\hline 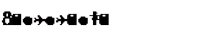 & ص & n. - & not & nase & manem \\
\hline 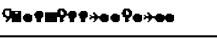 & 口 & مس & n & mone & שn \\
\hline 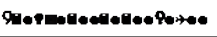 & 口 & nest & $n=\infty$ & mot & min \\
\hline 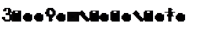 & - & $\mathbf{n} \times \mathbf{x}$ & nat & moren & merne \\
\hline 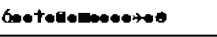 & पा & not & مس & men & שn: \\
\hline 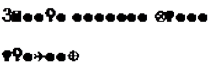 & प & x & nato & rnom & man \\
\hline 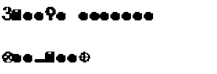 & n & nos. & $n=0$ & monem & $m$ \\
\hline 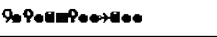 & $=$ & מח & nat & nasen & anocen \\
\hline Electloet+oe & $\square$ & ans- & nat- & - & - \\
\hline
\end{tabular}

\section{Results}

\subsection{Reliability and validity of scale analysis}

Reliability of each scale as measured by Cronbach's alpha is shown as Table 1. Most were over 0.7, except perceived mental support from others post-intervention. Spearman's rank-order correlation measured criterion-related validity with SDS as the criterion variable.

Note: a) Cronbach's alpha b) Criterion-related validity based on SDS as the criterion. Each scale was studied by correlation analysis.

$$
\mathrm{N}=26,{ }^{*} \mathrm{p}<.05 ; * * \mathrm{p}<.01
$$

\subsection{Change in $\operatorname{SIg} A$}

In general, changes in SIgA are associated with reduced physical ability and changes in mucosal tissue, as well as a weakened immune system [23]. Willemsen (2002) found that, among 78 males and 79 females, males show greater secretion of SIgA than females do during an active coping task [24] [25]. A large-scale epidemiological study of 2153 adults showed that males secrete more saliva as well as SIgA. However, this decreases with age, and lower socioeconomic status is associated with reduced saliva production [26]. Therefore, we considered potential bias from age and sex in the comparison between the SAT group (age range 34 to 60, 9 females) and the exercise group (age range 32 to 57, 9 females). Both the SAT group and the exercise group showed increased secretion of immunoglobulin SIgA, showing improved immunity (Figs. 1 and 2).
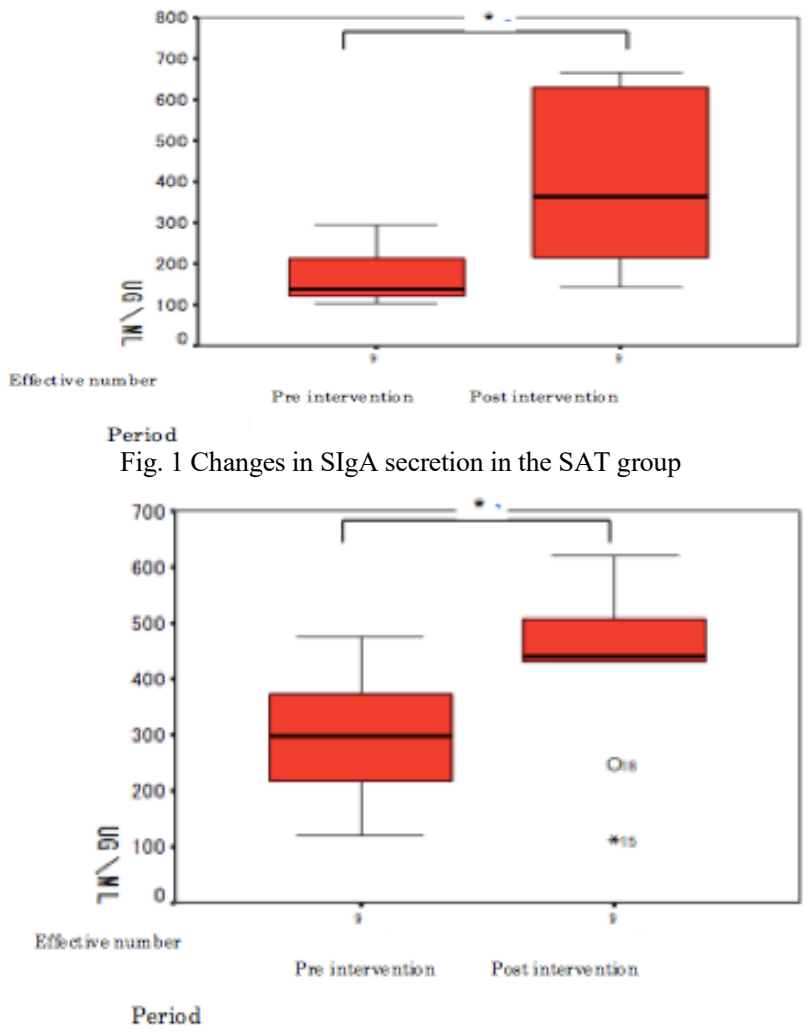

Fig. 2 Changes in SIgA secretion in the exercise group

Wilcoxon signed-rank test $(* \mathrm{p}=0.05) \mathrm{N}=9$

Pre-intervention - post-intervention $(z=-2.429, p=0.015)$

Wilcoxon signed-rank test $(* \mathrm{p}=0.05) \mathrm{N}=9$

Pre-intervention - post-intervention $(\mathrm{z}=-1.836, \mathrm{p}=0.012)$

\subsection{State anxiety and depression}


For the SAT group, a significant difference in depression and anxiety was observed between pre- and post-intervention and preintervention and the 1-month follow-up (Fig. 3), but there was no such difference between post-intervention and the 1-month followup. There were significant differences between pre- and postintervention and the 1-month follow-up, with sample size of 17 (all SAT program participants).

A significant difference in depression was also observed pre/post-intervention in the exercise group. However, there was no such difference at the 1-month follow-up, and no trend even at the $10 \%$ level. A significant difference in state anxiety was not observed pre-/post-intervention or between pre-intervention and the 1-month follow-up, nor was there a trend at the $10 \%$ level (Fig. 4)

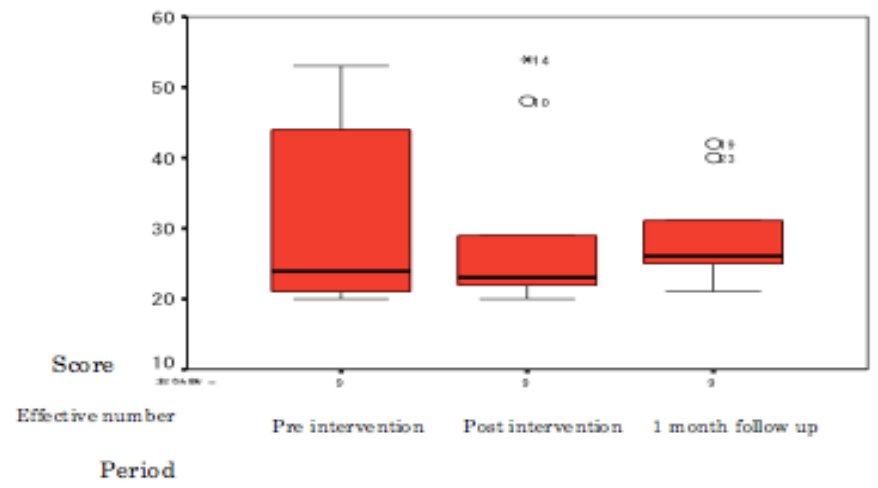

Fig. 3 Changes in anxiety and depression scales at pre- and post-intervention and the 1-month follow-up for the SAT group

Friedman test $\left(\chi^{2}=16.710, p<0.001\right) \mathrm{N}=9$

Wilcoxon signed-rank test, pre- - post-intervention $(z=-2.677, p=0.007)$; pre-intervention - 1-month follow-up $(z=-2.668, p=0.008)$

Bonferroni correction $*<.025$

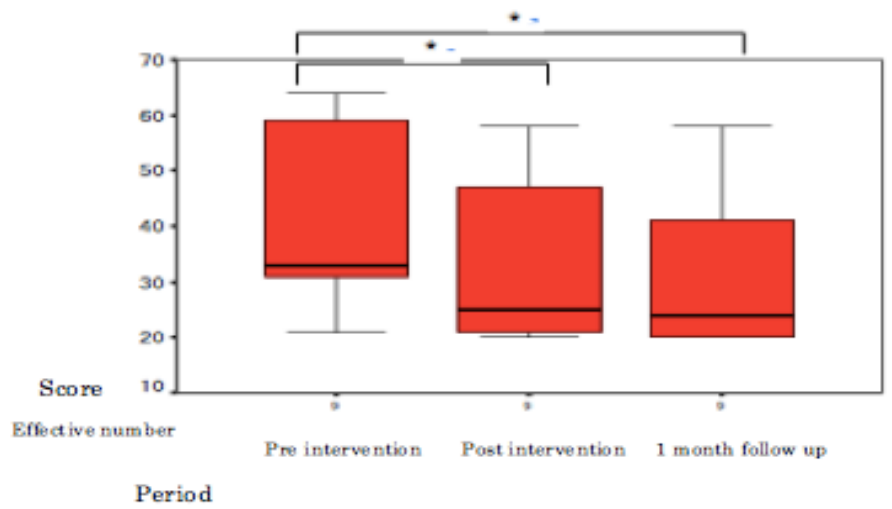

Fig. 4 Changes in state anxiety pre-/post-intervention and the 1-month follow-up in the exercise group

Friedman test $\left(\chi^{2}=0.800, \mathrm{p}=0.670\right) \mathrm{N}=9$

\subsection{Changes in self-image script}

In the SAT group, a significant difference in problem-solving behavior was observed between pre- and post-intervention and pre-intervention and the 1-month follow-up at the 5\% level (Fig 5). A significant difference in self-affirmation was observed between pre-intervention and 1-month follow-up (Fig. 6). A trend at the $10 \%$ level were observed for mental resilience and selfrepresentation. Significant differences in mental resilience and self-representation were observed for pre-/post-intervention and the 1-month follow-up at the $5 \%$ level.

In the exercise group, a significant difference in mental resilience and depression was observed pre-/post-intervention. At the 1-month follow-up, a significant difference was not observed, nor was there a trend at the $10 \%$ level. No significant differences were observed for self-affirmation, self-representation, problemsolving, or mental independence pre-/post-intervention or at the 1month follow-up, nor was there was trend at the $10 \%$ level.

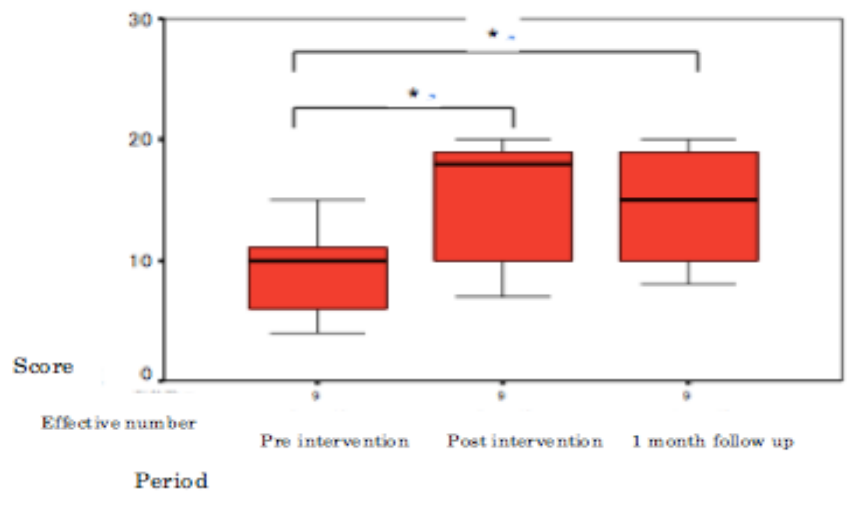

Fig. 5 Problem-solving changes in the SAT group

Friedman test $\left(\chi^{2}=9.750, \mathrm{p}=0.008\right) \mathrm{N}=9$

Wilcoxon signed-rank test, pre- - post-intervention $(z=-2.492, p=0.013)$; pre-intervention -1 -month follow-up $(z=-2.490, p=0.013)$

Bonferroni correction $*<.025$

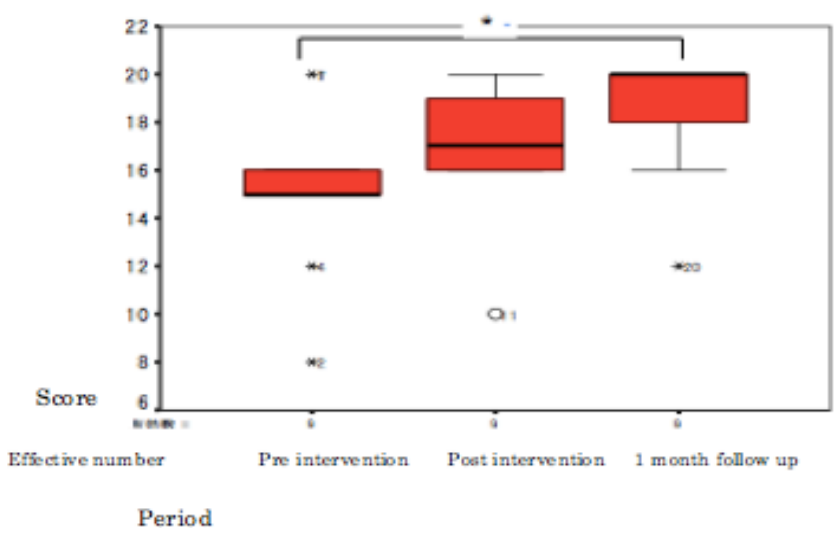

Fig. 6 Changes in self-affirmation for the SAT group

Friedman test $\left(\chi^{2}=11.655, \mathrm{p}=0.003\right) \mathrm{N}=9$

Wilcoxon signed-rank test, pre- - post-intervention $(z=-1.975, p=0.048)$; pre-intervention -1 -month follow-up $(\mathrm{z}=-2.374, \mathrm{p}=0.0176)$

Bonferroni correction $*<.025$

\subsection{Mental support from family}

A significant difference in both groups was observed, though this was a trend at the $10 \%$ level for the SAT group. A significant difference in mental support from family was observed at pre- and post-intervention and at the 1-month follow-up.

\section{Discussion}

We comparatively studied the SAT resilience program as the intervention group and an exercise group as the control group. We measured state anxiety, depression, and secretion of 


\section{Y. Kobayashi et al. / Advances in Science, Technology and Engineering Systems Journal Vol. 2, No. 2, 1-6 (2017)}

immunoglobulin in saliva (SIgA) at pre- and post-intervention. In the SAT group, a significant difference was observed in state anxiety, depression, and SIgA.

In the exercise group a significant difference was observed in depression and SIgA, excluding state anxiety. This result was similar to previous findings on cancer patients. One type of SAT imagery therapy, three-generation therapy (participants image their mother's womb and go back three generations, and then change their brief self-image by changing the image of crisis in the womb), developed in 2009, was carried out on cancer patients in the form of both group intervention and DVD intervention [27]. It showed that state anxiety and depression decreased significantly and SIgA increased significantly. It has also been reported that exercise increases SIgA secretion [28].

In this study, which used SAT imagery therapy, mental health and an immunity indicator (SIgA) improved between pre- and post-intervention. Changing the self-image can occur between preand post-intervention, a change particularly evident in problemsolving behavior. The intervention group had memories that their parent contacted their true self in terms of strictness, expression, and sadness. Therefore, they did not experience the feeling of being protected by the parent, and they had feelings of fear, anxiety, panic, self-hatred, and a sense of helplessness with regard to their true self. They even felt hopeless for the future. With daily observance of the surrogate facial representations of wished family, it is expected they would not feel fear, anxiety, self-hatred, panic, or helplessness.

Once the subjects engaged daily with the facial representation of wished family (SKP), their expectations of family decreased, and their family image improved. This has been shown in actual SKP therapy. It is thought that improving the family image, including parents and facial images of wished family (SKP), decreases feelings of fear, anxiety, and self-hatred, as well as improving self-image. Some benefits of this view of the self-image include, "it is less likely to get emotional," "able to judge while confirming each fact," and "try to make a forecast and plan before new initiatives." This facilitates the ability to forecast to solve the problem independently, helping to improve state anxiety and depression as well as immunity. A significant difference was observed in self-image, state anxiety, and self-affirmation in the SAT group from pre-intervention to 1-month. To compare with the exercise group, the SAT sample was reduced to 9. There was no difference at the $5 \%$ level, but there was a trend at the $10 \%$ level. For the entire SAT group (17 participants), there was a significant difference in self-affirmation, self-representation, and problemsolving, but not mental independence between pre-intervention and 1-month follow-up. State anxiety, depression, and mental resilience also significantly improved. The SAT resilience program improved mental health not only in the short term, but also 1 month later. Resilience developed into the strength to recover from stress, fight against stress, and improve. The selfimage script of self-affirmation, self-representation, problemsolving, and mental resilience remained. State anxiety and depression seemed to be prevented. However, there was not an appropriate control group for the 17 participants. Thus, the implications remain unclear. Previous research on nurses did not find a significant difference in the non-intervention group for a short-term intervention.

In a group intervention that applied SKP imagery therapy, characteristic anxiety decreased after 6 months. This was apparently caused by improvement of the self-image script, www.astesj.com reflecting self-value and characteristic behavior including problem-solving, self-repression, and emotion recognition [29]. Only self-affirmation changed significantly in a comparison of the assertion training group; however, in SKP imagery therapy, all self-image script factors - self-value and characteristic behavior including depression and problem-solving - changed significantly [30]. Given these considerations, the findings should extend to the general population. Further research with larger control groups is required. This appears to be a sustainable effect, however, as a short-term intervention of company management improved characteristic behaviors including self-repression, anxiety, and depression up to a 6-month follow-up [31].

This study was an intervention study of resilience among the general population on their resistance to daily stresses. Because of the earthquake in eastern Japan, some of the participants might have been under unusual stresses. Regarding this situation, we should consider individual intervention. Several issues remain for future study. Sample size should be increased for both groups. Further, the SAT therapy should be extended beyond the simple SAT method to the clinical three-generation therapy method, even for the short term. Therefore, to apply three-generation therapy, development is required to be appropriate for group work and sustainable effects. We will consider developing the short program as well as a 3-day training program moving forward.

\section{References}

[1] Ministry of Health, Labour and Welfare: A minister's secretariat Statistical Information Department society statistics section life of the people basics investigation room, http://www.mhlw.go.jp/toukei/saikin/hw/k-tyosa/ktyosa10/, 12/12, 2011

[2] National Police Agency: Life security station, Life security planning division, http://www.npa.go.jp/safetylife/seianki/H22jisatsunogaiyou.pdf,12/12, 2011

[3] Rutter, M: Resilience in the face of adversity, protective factors and resistance to psychiatric disorder. Br J Psychiatry 147: 598-611, 1985

[4] Herman, J L: Trauma and recovery, Misuzu Publishing, Tokyo, 283-294, 1999

[5] Hoge, E A, Austin, E D, Pollack, M H: Resilience: research evidence and conceptual considerations for posttraumatic stress disorder. Depress Anxiety, 24(2), 139-152, 2007

[6] Bonanno, G A: Loss, trauma, and human resilience: have we underestimated the human capacity to thrive after extremely aversive events? Am Psychol, 59(1), 20-28, 2004

[7] Naoi, K: Analysis of local mental health activity earthquake disaster three and a half months after the Niigata Chuetsu Earthquake and 13 months later: findings and the PTSD risk factor, Jpn Soc Psychiatry Mag, 67(4), 486, 2009

[8] Munakata, T: Structured Association Technique Therapy, Kaneko Bookshop, Tokyo, 35-32, 2006

[9] Masten, A S and Coatsworth, J D: The development of competence in favorable and unfavorable environments-lessons from research on successful children, Am Psychol, 53, 205-220, 1998

[10] D'Zurilla, T J and Nezu, A M: Development and preliminary evaluation of the Social Problem-Solving Inventory, Psychol Assess, 2, 159-163, 1990

[11] Werner, E E: Protective factors and individual resilience. Handbook of Early Childhood Intervention. Ed. By Meisel S J and Shonkoff, J P, Cambridge University Press, Cambridge, 97-116, 1990

[12] Wagnild, G M and Young, H M: Development and psychometric evaluation of the Resilience Scale, J Nurs Meas, 1, 165-178, 1993

[13] Oshio, A et al.: Construct validity of the Adolescent Resilience Scale. Psychol Rep, 93, 1217-1222, 2003

[14] Munakata, T: Measure the unsolved feelings that became the scientific potentiality of remembrance, transmission, the conversion of the heart, Health Couns, 3(6), 94-102, 2001 
[15] Munakata, T: Recognition and coping action of the stress source, Mental Health Study, National Mind Nerve Center, 39, 29-40, 1993

[16] Kazuhiro, Y and Munakata, T: Development of the mental health-related standard for Japanese, Soc Mental Health, 3, 63-67, 1997

[17] Munakata, T et al.: Stress and mind health degree of the city citizen, 32, 4968,1986

[18] Zung, W W K: A Self-Rating Depression Scale. Arch Gen Psychiatry, 12, 6370,1973

[19] Fukuda, K and Kobayashi, S: Japanese edition SDS use guide, 30 Quadrillion Bunches, Kyoto, 1970

[20] Spielberger, C D et al: STAI manual, Palo Alto, CA, Consulting Psychologist Press, 1970

[21] Hidano, $\mathrm{T}$ et al.: New publication STAI manual, Business Education Publication, Tokyo, 1-10, 2000

[22] Arita, H: Where rhythm movement lets serotonin nervous system activate from basic medicine, Japanese Medical Affairs Courier, 4453, 38-42, 2009

[23] Sawada, et al.: Change of the secretion type immunoglobulin A out of saliva in the everyday life of the long-term medical treatment elderly person, Sports Sci, 57, 241-248, 2008

[24] Willemsen, G, et al.: Cellular and mucosal immune reactions to mental and cold stress: associations with gender and cardiovascular reactivity, Psychophysiol, 39, 222-228, 2002

[25] Fujiwara, S and Yogo, M: Stress and affective psychology organon with sIgA as an index, Doshisha Psychol, 50, 57-81, 2003

[26] Evans, P et al.: Social class, sex, and age difference in mucosal immunity in a large community sample, Brain, Behav Immun, 14, 41-48, 2000

[27] Higuchi, $\mathrm{N}$ et al.: Comparative examination of the effects of the SAT-DVD learning program therapy and the Qigong (the traditional Chinese breathing exercise) therapy to alleviate stress in cancer survivors, Int J Struct Assoc Technique, 3, 61-90, 2009

[28] Akama, $\mathrm{T}$ et al.: Change of salivary secretory $\operatorname{IgA}$ by 42 months exercise training in elderly persons, Sports Health Sci, 2, 122-127, 2005

[29] Taniguchi, S: Group intervention support about mental health and resilience of the nurse, J Health Counsel, 18, 19-26, 2012

[30] Taniguchi, S: Middle effect of the mental health by the self-image rebuilding using the image method to a nurse, Jpn Health Med Care Behav Sci Soc Mag, 28(1), 71-81, 2013

[31] Yamamoto, $\mathrm{M}$ and Munakata, T: Examination of self-image and -9 selfreward type carrier training back issue intervention effects of the company manager, Sociol Mental Health, 17, 31-38, 2011 